Provided for non-commercial research and education use. Not for reproduction, distribution or commercial use.

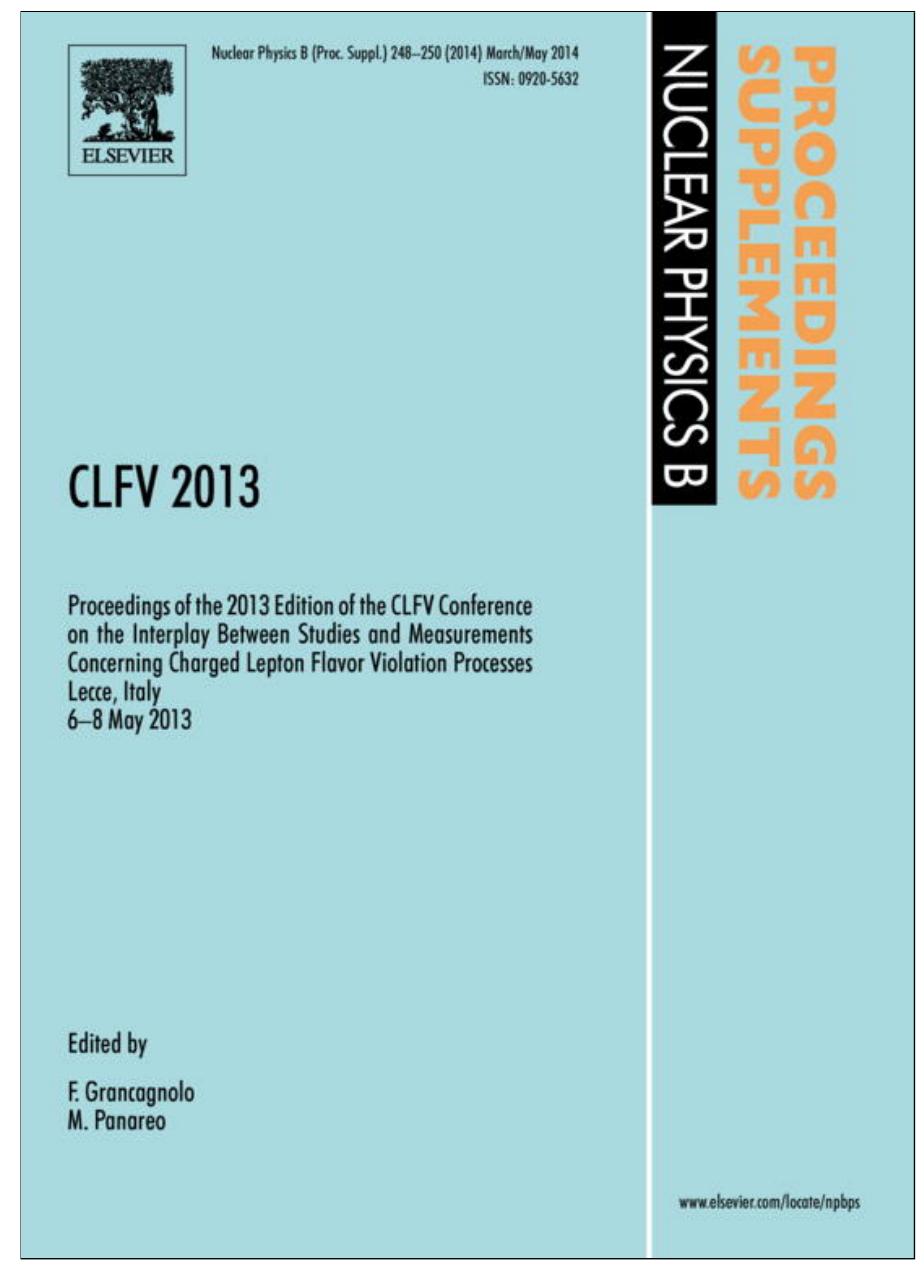

This article appeared in a journal published by Elsevier. The attached copy is furnished to the author for internal non-commercial research and education use, including for instruction at the authors institution and sharing with colleagues.

Other uses, including reproduction and distribution, or selling or licensing copies, or posting to personal, institutional or third party websites are prohibited.

In most cases authors are permitted to post their version of the article (e.g. in Word or Tex form) to their personal website or institutional repository. Authors requiring further information regarding Elsevier's archiving and manuscript policies are encouraged to visit:

http://www.elsevier.com/authorsrights 


\title{
Charged lepton flavor violation beyond minimal supersymmetry
}

\author{
A. Vicente \\ IFPA, Dep. AGO, Université de Liège, Bat B5, Sart-Tilman B-4000 Liège 1, Belgium
}

\begin{abstract}
We discuss charged lepton flavor violation in supersymmetric models with extended leptonic sectors at low energies. Contrary to the usual high-scale realizations of the seesaw mechanism, these non-minimal supersymmetric models have new superfields and/or interactions at the supersymmetric scale. As a consequence of this, the resulting lepton flavor violating phenomenology may be very different from that of minimal models.
\end{abstract}

Keywords: supersymmetry, leptons, flavor, R-parity violation

\section{Introduction}

Since the discovery of neutrino oscillations, the violation of lepton flavor is a well-established fact. However this behavior has never been observed in processes involving charged leptons. This is well understood in the Standard Model (SM) minimally extended to include neutrino masses. Since the only source of lepton flavor violation (LFV) is the neutrino mass matrix itself, all LFV processes are highly suppressed. This makes the observation of charged lepton flavor violation a clear sign of new physics.

New sources of LFV can be found in most extensions of the leptonic sector. These may be caused by new interactions (renormalizable or non-renormalizable) or by entire new sectors coupled to the charged leptons. In the case of supersymmetry (SUSY), the new degrees of freedom provided by the superpartners of the SM leptons have potentially large contributions to LFV processes, leading to stringent constraints on the slepton masses and mixings.

Most papers on SUSY phenomenology focus on minimal models, such as the Minimal Supersymmetric Standard Model (MSSM) ${ }^{1}$. However, neutrinos are massless in the MSSM and thus the leptonic sector must

Email address: Avelino.Vicente@ulg.ac.be (A. Vicente)

${ }^{1}$ For a recent work on LFV in the MSSM see [1]. be further extended in order to account for the observed neutrino masses and mixings. In general, this can be done in two different ways:

- High-energy extensions: In this family of models the new degrees of freedom responsible for neutrino masses live at very high energy scales. Therefore, the physics at the SUSY scale is well described by the MSSM and the effect of the heavy fields is induced by Renormalization Group Equations running.

- Low-energy extensions: In this family of models the new degrees of freedom are present at low energies. These include new particles and/or interactions. Their effect on the phenomenology is direct, as they fully participate at the SUSY scale.

The first category can be seen as Minimal SUSY, since the low-energy theory is just the MSSM. These are the most studied SUSY neutrino mass models in the literature and the simplest example is the classical SUSY seesaw $^{2}$. On the contrary, models belonging to the second category can be seen as non-minimal SUSY. Here

\footnotetext{
${ }^{2}$ By classical SUSY seesaw we mean the usual realization of the seesaw mechanism where the seesaw messengers have masses close to the unification scale.
} 

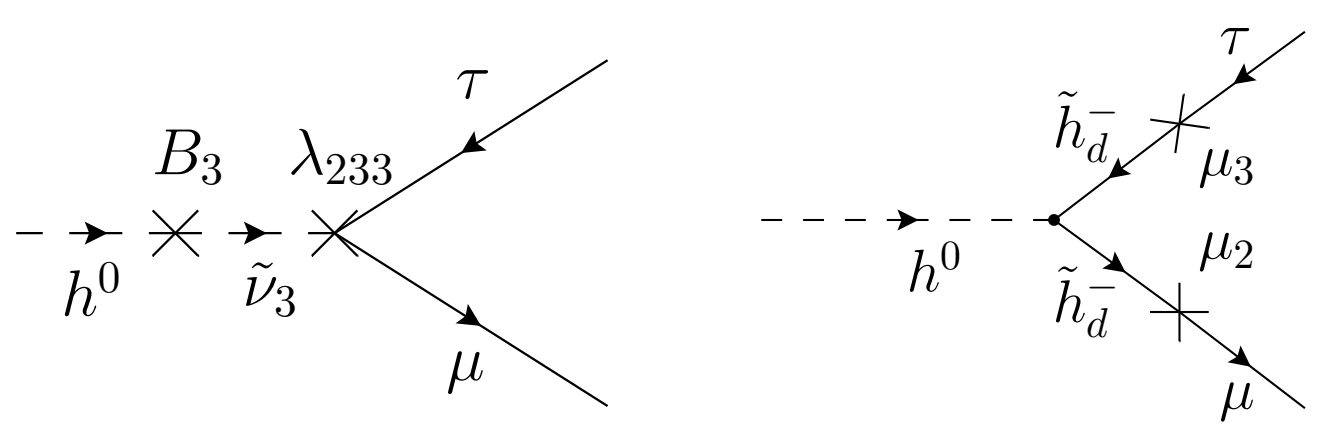

Figure 1: Tree-level R-parity violating contributions to $h \rightarrow \mu \tau$. On the left, a $B \lambda$ contribution. On the right, a $\mu_{i}^{2}$ contribution. Figure borrowed from [2].

we will concentrate on this case and discuss some recent works related to non-minimal extensions of the leptonic sector in SUSY models. As will be clear from the following examples, non-minimal SUSY models may have a very different LFV phenomenology from that of minimal ones.

\section{R-parity violating models}

R-parity violation (RPV) is usually regarded as a dangerous possibility due to the non-observation of proton decay. For that reason, most SUSY models simply assume this $a d-h o c$ symmetry that forbids all renormalizable baryon $(B)$ and lepton $(L)$ number violating operators. However, there is no need to forbid all these operators. In fact, in order to stabilize the proton it is sufficient to get rid of just one of these terms, namely the baryon number violating one.

Furthermore, the presence of R-parity violating couplings leads to a much richer collider phenomenology due to the decay of the lightest supersymmetric particle (LSP). This adds a further step in the SUSY decay chains at the LHC and changes dramatically the resulting signatures $[3,4]$. This has also been recently considered in order to relax the stringent bounds on the squark and gluino masses, otherwise increased to values beyond expectations based on naturalness arguments, see for example $[5,6]$.

In what concerns the leptonic sector, the lepton number violating interactions induce Majorana neutrino masses $[7,8]$. These models are thus a very economical framework to explain neutrino masses and mixings and, as we will discuss below, usually have many nonstandard LFV signatures.
Finally, the tight connection between neutrino masses and the decay of the LSP allows us to have a direct probe for a general class of these models (those where the relevant $\mathrm{L}$ violating operator is the bilinear term $\mu_{i} \widehat{L}_{i} \widehat{H}_{u}$ ) at the LHC, see the recent Ref. [9].

\subsection{Higgs boson LFV decays}

After the recent discovery of the long-awaited Higgs boson, the focus has shifted towards the experimental determination of its properties. On the one hand, they are crucial to conclude that the particle discovered at the LHC actually corresponds to the Higgs boson, the footprint left by the spontaneous breaking of the SM gauge symmetry. On the other hand, even if this new particle turns out be a very SM-like Higgs boson, there is still hope for some non-standard properties.

The LFV decay $h \rightarrow \ell_{i} \ell_{j}$, with $i \neq j$, has recently got some attention $[10,11]$. As shown in [10], in the case of final states including $\tau$ leptons, LHC data can already put constraints similar to those from low-energy experiments. Therefore, the question is what type of models allow for large Higgs LFV branching ratios and whether these are compatible with the usual low-energy constraints.

This question was recently addressed in the context of R-parity violating models in $[2,12]$. The authors of these references considered the MSSM extended to include all renormalizable (L violating) RPV couplings and computed $\operatorname{Br}(h \rightarrow \mu \tau)$ at the 1-loop level. The key point in RPV models is the particles-sparticles mixing induced by the RPV parameters and how it leads to treelevel LFV Higgs decays.

Two examples are shown in figure 1. On the lefthand side, a $B \lambda$ contribution is shown, whereas on the right-hand side a $\mu_{i}^{2}$ contribution is presented. Here $\mu_{i}$ 


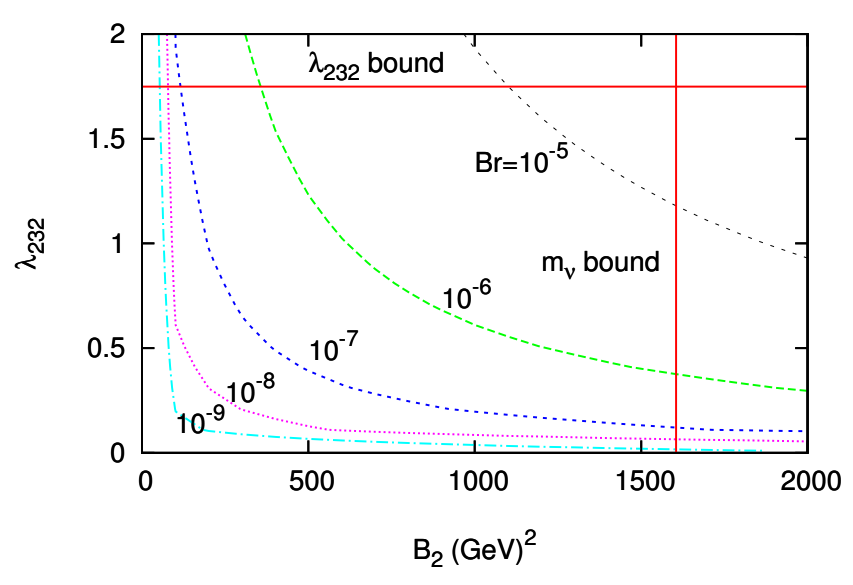

Figure 2: $\operatorname{Br}(h \rightarrow \mu \tau)$ contours on the $B_{2}-\lambda_{232}$ plane. The continuous horizontal and vertical lines show approximate limits due to neutrino masses (in case of $B_{2}$ ) and charged current experiments (in case of $\left.\lambda_{232}\right)$. Figure borrowed from [2].

and $\lambda_{i j k}$ are the superpotential couplings $\mathcal{W} \supset \mu_{i} \widehat{L}_{i} \widehat{H}_{u}+$ $\frac{1}{2} \lambda_{i j k} \widehat{L}_{i} \widehat{L}_{j} \widehat{e}_{k}^{c}$ and $B_{i}$ is the soft SUSY breaking parameter $\mathcal{L} \supset B_{i} \tilde{L}_{i} H_{u}{ }^{3}$.

The parameter combination that gives rise to the largest $\operatorname{Br}(h \rightarrow \mu \tau)$ is $B \lambda$, where the mixing between the Higgs boson and the sneutrinos is combined with the lepton number violating $\lambda$ coupling. However, the same combination of couplings contributes to neutrino masses $[13,14,15]$. Moreover, the $\lambda$ coupling is constrained by charged current experiments [16]. For these reasons, the largest value for $\operatorname{Br}(h \rightarrow \mu \tau)$ compatible with the previous bounds is actually found to be quite small. This is shown in figure 2, where several $\operatorname{Br}(h \rightarrow \mu \tau)$ contours are drawn on the $B_{2}-\lambda_{232}$ plane. From this figure one can easily conclude that $\operatorname{Br}(h \rightarrow \mu \tau)$ can reach, at most, a few $\times 10^{-5}$.

Although this result is a little disappointing (the LHC sensitivity with $20 \mathrm{fb}^{-1}$ at $8 \mathrm{TeV}$ was estimated in [11] to be around $\mathrm{Br} \sim 10^{-3}$ ), further investigation in extended RPV scenarios might reveal better chances.

\subsection{Exotic muon decays}

A very interesting framework for lepton flavor violation is that of spontaneous R-parity violation. In this case, and in order to establish a bridge to neutrino masses, one considers a supersymmetric theory that in

\footnotetext{
${ }^{3}$ We are following here the notation in $[2,12]$. The $\mu_{i}$ parameters should not be confused with the usual Higgs superpotential mass term $\mu$.
}

principle conserves lepton number, but whose vacuum breaks $\mathrm{it}^{4}$. If the breaking is caused by the vacuum expectation value (VEV) of a scalar field with an odd lepton number, R-parity is broken as well.

The main difference with respect to explicit R-parity violation is the existence of a massless Goldstone boson, the Majoron $(J)[17,18]$, associated to the spontaneous breaking of the continuous $U(1)_{L}$ symmetry. The nature of this massless state is actually of great importance for the phenomenological success of the model. The first attemp in this direction was made in [19], where the breaking of R-parity was caused by the VEV of a lefthanded sneutrino. The model was eventually excluded since the doublet nature of the majoron leads to conflict with LEP bounds and astrophysical data [20, 21]. However, more refined models, where the violation of lepton number is induced by a gauge singlet, are still valid possibilities. We will consider here the model introduced in [22].

The model under consideration [22] contains three additional singlet superfields, namely, $\widehat{v}^{c}, \widehat{S}$ and $\widehat{\Phi}$, with lepton number assignments of $L=-1,1,0$ respectively. The superpotential can be written as

$$
\begin{aligned}
W & =Y_{u}^{i j} \widehat{Q}_{i} \widehat{u}_{j}^{c} \widehat{H}_{u}+Y_{d}^{i j} \widehat{Q}_{i} \widehat{d}_{j} \widehat{H}_{d}+Y_{e}^{i j} \widehat{L}_{i} \widehat{e}_{j}^{c} \widehat{H}_{d} \\
& +Y_{v}^{i} \widehat{L}_{i} \widehat{v}^{c} \widehat{H}_{u}-h_{0} \widehat{H}_{d} \widehat{H}_{u} \widehat{\Phi}+h \widehat{\Phi} \widehat{\varphi}^{c} \widehat{S}+\frac{\lambda}{3 !} \widehat{\Phi}^{3} .
\end{aligned}
$$

For simplicity we consider only one generation of $\widehat{v}^{c}$ and $\widehat{S}$. Adding more generations of $\widehat{v}^{c}$ or $\widehat{S}$ does not add any qualitatively new features to the model. At low energy, i.e. after electroweak symmetry breaking, various fields acquire VEVs. Besides the usual MSSM Higgs boson VEVs $v_{d}$ and $v_{u}$, these are $\langle\Phi\rangle=v_{\Phi} / \sqrt{2},\left\langle\tilde{v}^{c}\right\rangle=v_{R} / \sqrt{2}$, $\langle\tilde{S}\rangle=v_{S} / \sqrt{2}$ and $\left\langle\tilde{v}_{i}\right\rangle=v_{i} / \sqrt{2}$. Note, that $v_{R} \neq 0$ generates effective bilinear terms $\mu_{i}=Y_{v}^{i} v_{R} / \sqrt{2}$ and that $v_{R}, v_{S}$ and $v_{i}$ violate lepton number as well as R-parity.

Although the presence of a massless majoron is allowed by the experimental contraints, it dramatically changes the phenomenology both at collider and lowenergy experiments $[23,24]$. In particular, it leads to new LFV processes, such as $\mu \rightarrow e J$ or $\mu \rightarrow e J \gamma$. The exotic decay $\mu \rightarrow e J$ was first studied in [25] and later revisited in [24], where the decay with an additional photon was also considered.

The decays $\ell_{i} \rightarrow \ell_{j} J$ can be calculated from the general coupling $\chi_{i}^{+}-\chi_{j}^{-}-P_{k}^{0}$. A straightforward computation [24] shows that the $e-\mu-J$ coupling, $O_{e \mu J}$, is of the form $O_{e \mu \mathrm{J}} \sim \frac{1}{v_{R}} \times \mathrm{RPV}$ parameters, which

\footnotetext{
${ }^{4}$ One could also think of spontaneous baryon number violation, but that is clearly out of the scope of this talk.
} 
makes us conclude that, in general, one expects large partial decay widths to majorons if $v_{R}$ is low. However, searches for $\mu \rightarrow e J$ have to deal with a huge irreducible background coming from the standard muon decay $\mu \rightarrow e v v$. Moreover, there are no experiments looking for $\mu \rightarrow e J$ and the current limit on the branching ratio, $\operatorname{Br}(\mu \rightarrow e J) \lesssim 10^{-5}$, dates back to $1986[26]^{5}$. For these reasons, we consider the decay $\mu \rightarrow e J \gamma$ which might be more interesting due to the existent experiments looking for $\mu \rightarrow e \gamma$.

It is easy to find a very simple relation between the two branching ratios

$$
\operatorname{Br}(\mu \rightarrow e J \gamma)=\frac{\alpha}{2 \pi} \mathcal{I}\left(x_{\text {min }}, y_{\text {min }}\right) \operatorname{Br}(\mu \rightarrow e J) .
$$

Here $\mathcal{I}\left(x_{\min }, y_{\min }\right)$ is a phase space integral given by

$$
\mathcal{I}\left(x_{\min }, y_{\min }\right)=\int d x d y \frac{(x-1)(2-x y-y)}{y^{2}(1-x-y)},
$$

the dimensionless parameters $x, y$ are defined as usual

$$
x=\frac{2 E_{e}}{m_{\mu}} \quad, \quad y=\frac{2 E_{\gamma}}{m_{\mu}}
$$

and $x_{\min }$ and $y_{\min }$ are the minimal electron and photon energies measured in a given experiment.

The question now is whether the MEG experiment can look for this process. Figure 3 shows the value of the phase space integral $\mathcal{I}\left(x_{\min }, y_{\min }\right)$ as a function of $x_{\min }$ for three different values of $y_{\min }$ and for two choices of $\cos \theta_{e \gamma}$. The MEG proposal describes the cuts used in the search for $\mu \rightarrow e \gamma$ as $x_{\text {min }} \geq 0.995, y_{\text {min }} \geq 0.99$ and $\left|\pi-\theta_{e \gamma}\right| \leq 8.4 \mathrm{mrad}$. For these values one finds $I \simeq 6 \cdot 10^{-10}$. A limit for $\operatorname{Br}(\mu \rightarrow e \gamma)$ of $\operatorname{Br}(\mu \rightarrow e \gamma) \leq$ $10^{-13}$ then translates into a limit of $\operatorname{Br}(\mu \rightarrow e J) \leq 0.14$, obviously meaningless. To improve upon this bound, it is necessary to relax the cuts. For example, relaxing the cut on the opening angle to $\cos \theta_{e \gamma}=-0.99$, the value of the integral increases by more than 3 orders of magnitude for $x_{\min }=y_{\min } \geq 0.95$.

On the other hand, such a change in the analysis is prone to induce background events, which the MEG cuts were designed for to avoid. In particular, accidental background from muon annihilation in flight ${ }^{6}$. Therefore, although one could in principle increase the value

\footnotetext{
${ }^{5}$ In fact, the bound we provide here is based on a reinterpretation of the results of [26], which considered a slightly different scenario.

${ }^{6}$ Less important is the irreducible background induced by prompt events from the standard model radiative decay $\mu \rightarrow e v v \gamma$. Moreover, kinematical information might allow to discriminate this decay from $\mu \rightarrow e J \gamma$
}

of the phase space integral $\mathcal{I}\left(x_{\min }, y_{\min }\right)$, the background in that case would make the search for a positive signal impossible. Certainly, a better timing resolution of the experiment would be required to reduce this background.

\section{Supersymmetric low-scale seesaw models}

Let us now briefly discuss LFV in low-scale seesaw models. For more information about the phenomenology in non-SUSY models see [27].

The most popular way to address neutrino masses is the famous seesaw mechanism. In its traditional form, the seesaw mechanism explains the smallness of neutrino masses thanks to the suppression by a high scale, $\Lambda$, at which neutrino masses are generated. Several realizations (supersymmetric or not) of this framework are known. In the supersymmetric versions of these realizations, the low energy spectrum is composed by the MSSM superfields, since the seesaw messengers decouple at $\Lambda \gg m_{S U S Y}$.

At the SUSY scale, the misalignment of the slepton mass matrices with respect to those of the SM charged leptons induces lepton flavor violation. However, different LFV processes may have very different rates. The classical supersymmetric seesaw usually predicts that the branching ratios for the decays $\ell_{i} \rightarrow 3 \ell_{j}$ are roughly a factor $\alpha$ smaller than those for the corresponding decays $\ell_{i} \rightarrow \ell_{j} \gamma[28,29,30]$,

$$
\operatorname{Br}\left(\ell_{i} \rightarrow 3 \ell_{j}\right) \simeq \frac{\alpha}{3 \pi}\left(\log \left(\frac{m_{\ell_{i}}^{2}}{m_{\ell_{j}}^{2}}\right)-\frac{11}{4}\right) \operatorname{Br}\left(\ell_{i} \rightarrow \ell_{j} \gamma\right) .
$$

Therefore, in these scenarios, which belong to the minimal SUSY category, the most relevant LFV process is $\ell_{i} \rightarrow \ell_{j} \gamma$.

The relation in Eq. (5) can be traced back to the socalled dipole dominance in high-scale seesaw models. Among all contributions to $\ell_{i} \rightarrow 3 \ell_{j}$, the dipole generated in photon penguins is the dominant one. For this reason, the branching ratios for $\ell_{i} \rightarrow \ell_{j} \gamma$ and $\ell_{i} \rightarrow 3 \ell_{j}$ turn out to be proportional. However, the dipole dominance can be broken in non-minimal scenarios that include new superfields besides those in the MSSM. This is in fact the case of supersymmetric low-scale seesaw models.

Contrary to the classical SUSY seesaw, in low-scale seesaw models the new states (in addition to those of the MSSM) do not decouple: indeed, the seesaw messengers can be as light as to be present at the SUSY scale, leading to new contributions that can change the 

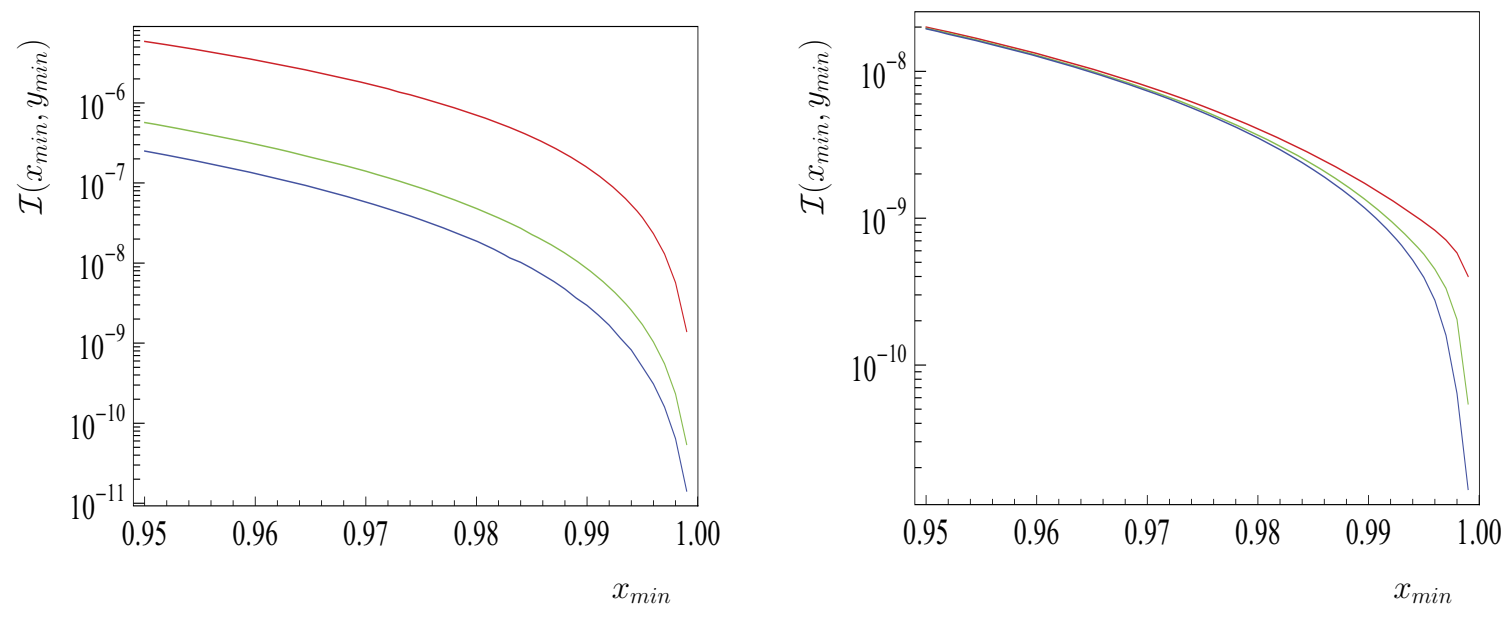

Figure 3: The phase space integral for the decay $\mu \rightarrow e J \gamma$ as a function of $x_{\min }$ for three different values of $y_{\min }=0.95,0.99,0.995$ from top to bottom and for two different values of $\cos \theta_{e \gamma}$. To the left $\cos \theta_{e \gamma}=-0.99$, to the right $\cos \theta_{e \gamma}=-0.99997$.

picture dramatically ${ }^{7}$. This has recently fuelled a significant number of studies. Let us now mention some of them:

- Non-SUSY boxes: Several authors have recently studied LFV in the presence of light right-handed neutrinos, paying special attention to $\ell_{i} \rightarrow 3 \ell_{j}$ and $\mu-e$ conversion in nuclei $[32,33,34,35]$. Surprisingly, it has been found that the main contributions to these two processes may come from (non-supersymmetric) box diagrams. These nonSUSY contributions tend to dominate for light right-handed neutrinos. Therefore, although they are not genuine SUSY contributions, they must be taken into account in any SUSY study.

- Z-penguins: The presence of light 'right-handed' sneutrinos also gives rise to new contributions. In [36] it was pointed out that in this case the Zpenguins, usually regarded as subdominant, can in fact provide the dominant contributions to LFV processes such as $\ell_{i} \rightarrow 3 \ell_{j}$ and $\mu-e$ conversion in nuclei. This was further explored in [37, 38, 39], where enhancements to the abovementioned processes thanks to the supersymmetric Z-penguins were found in several contexts.

\footnotetext{
${ }^{7} \mathrm{~A}$ prime example of this class of models is the inverse seesaw [31]. The inverse seesaw can be embedded in the MSSM by the addition of two extra gauge singlet superfields with opposite lepton numbers $(+1$ and -1$)$. In this framework one can in principle have large neutrino Yukawa couplings compatible with a seesaw scale close to the SUSY one.
}

In both cases, the relation in Eq. (5) is not fulfilled and one can actually have $\ell_{i} \rightarrow 3 \ell_{j}$ and $\mu-e$ conversion in nuclei as the most constraining observables.

\section{Final remarks}

In this talk we have reviewed some recent developments in lepton flavor violation in non-minimal SUSY models. The bottom line is that lepton flavor violation may be much richer than expected when one goes to non-minimal supersymmetric extensions of the leptonic sector. In most models the common lore (established in the MSSM) turns out to be completely wrong, and thus specific studies must be performed in order to correctly describe the corresponding phenomenology. This in fact translates into two messages: (1) for the theorists, lepton flavor violation might be much more intricate than what minimal models predict. We should be open-minded and consider non-standard scenarios where the leptonic sector is extended beyond the MSSM realization. And (2) for the experimentalists, although minimal models are well motivated, lepton flavor violation might show up in non-standard channels. We must be ready to find a signal there as well.

\section{References}

[1] M. Arana-Catania, S. Heinemeyer, M. Herrero, New Constraints on General Slepton Flavor Mixing, Phys.Rev. D88 (2013) 015026. arXiv:1304.2783, doi:10.1103/PhysRevD.88.015026.

[2] A. Arhrib, Y. Cheng, O. C. Kong, Higgs to mu+tau Decay in Supersymmetry without R-parity, Europhys.Lett. 101 (2013) 31003. arXiv:1208.4669, doi:10.1209/0295-5075/101/31003. 
[3] H. Dreiner, F. Staub, A. Vicente, W. Porod, General MSSM signatures at the LHC with and without Rparity, Phys.Rev. D86 (2012) 035021. arXiv:1205.0557, doi:10.1103/PhysRevD.86.035021.

[4] H. Dreiner, F. Staub, A. Vicente, General NMSSM signatures at the LHC, Phys.Rev. D87 (3) (2013) 035009. arXiv:1211.6987, doi:10.1103/PhysRevD.87.035009.

[5] P. W. Graham, D. E. Kaplan, S. Rajendran, P. Saraswat, Displaced Supersymmetry, JHEP 1207 (2012) 149. arXiv:1204.6038, doi:10.1007/JHEP07(2012)149.

[6] M. Hanussek, J. Kim, Testing neutrino masses in the Rparity violating minimal supersymmetric standard model with LHC results, Phys.Rev. D85 (2012) 115021. arXiv:1205.0019, doi:10.1103/PhysRevD.85.115021.

[7] L. J. Hall, M. Suzuki, Explicit R-Parity Breaking in Supersymmetric Models, Nucl.Phys. B231 (1984) 419. doi:10.1016/05503213(84)90513-3.

[8] G. G. Ross, J. Valle, Supersymmetric Models Without R-Parity, Phys.Lett. B151 (1985) 375. doi:10.1016/0370-2693(85)916582.

[9] F. de Campos, O. Eboli, M. Magro, W. Porod, D. Restrepo, et al., Probing Neutralino Properties in Minimal Supergravity with Bilinear R-Parity Violation, Phys.Rev. D86 (2012) 075001. arXiv:1206.3605, doi:10.1103/PhysRevD.86.075001.

[10] R. Harnik, J. Kopp, J. Zupan, Flavor Violating Higgs Decays, JHEP 1303 (2013) 026. arXiv:1209.1397, doi:10.1007/JHEP03(2013)026.

[11] S. Davidson, P. Verdier, LHC sensitivity to the decay $h \rightarrow$ $\tau^{ \pm} m u^{\mp}$, Phys.Rev. D86 (2012) 111701. arXiv:1211.1248, doi:10.1103/PhysRevD.86.111701.

[12] A. Arhrib, Y. Cheng, O. C. Kong, A Comprehensive Analysis on Lepton Flavor Violating Higgs to $\mu \bar{\tau}+\tau \bar{m} u$ Decay in Supersymmetry without R Parity, Phys.Rev. D87 (2013) 015025. arXiv:1210.8241, doi:10.1103/PhysRevD.87.015025.

[13] Y. Grossman, H. E. Haber, (S)neutrino properties in Rparity violating supersymmetry. 1 . CP conserving phenomena, Phys.Rev. D59 (1999) 093008. arXiv:hep-ph/9810536, doi:10.1103/PhysRevD.59.093008.

[14] E. J. Chun, S. K. Kang, One loop corrected neutrino masses and mixing in supersymmetric standard model without Rparity, Phys.Rev. D61 (2000) 075012. arXiv:hep-ph/9909429, doi:10.1103/PhysRevD.61.075012.

[15] S. Davidson, M. Losada, Neutrino masses in the $R(p)$ violating MSSM, JHEP 0005 (2000) 021. arXiv:hep-ph/0005080.

[16] R. Barbier, C. Berat, M. Besancon, M. Chemtob, A. Deandrea, et al., R-parity violating supersymmetry, Phys.Rept. 420 (2005) 1-202. arXiv:hep-ph/0406039, doi:10.1016/j.physrep.2005.08.006.

[17] Y. Chikashige, R. N. Mohapatra, R. Peccei, Are There Real Goldstone Bosons Associated with Broken Lepton Number?, Phys.Lett. B98 (1981) 265. doi:10.1016/0370-2693(81)900113.

[18] G. Gelmini, M. Roncadelli, Left-Handed Neutrino Mass Scale and Spontaneously Broken Lepton Number, Phys.Lett. B99 (1981) 411. doi:10.1016/0370-2693(81)90559-1.

[19] C. Aulakh, R. N. Mohapatra, Neutrino as the Supersymmetric Partner of the Majoron, Phys.Lett. B119 (1982) 136. doi:10.1016/0370-2693(82)90262-3.

[20] G. G. Raffelt, Stars as laboratories for fundamental physics: The astrophysics of neutrinos, axions, and other weakly interacting particles. Chicago, USA: Univ. Pr. 664 p.

[21] C. Amsler, et al., Review of Particle Physics, Phys.Lett. B667 (2008) 1-1340. doi:10.1016/j.physletb.2008.07.018.

[22] A. Masiero, J. Valle, A Model for Spontaneous R Parity Breaking, Phys.Lett. B251 (1990) 273-278. doi:10.1016/0370-
2693(90)90935-Y.

[23] M. Hirsch, A. Vicente, W. Porod, Spontaneous R-parity violation: Lightest neutralino decays and neutrino mixing angles at future colliders, Phys.Rev. D77 (2008) 075005. arXiv:0802.2896, doi:10.1103/PhysRevD.77.075005.

[24] M. Hirsch, A. Vicente, J. Meyer, W. Porod, Majoron emission in muon and tau decays revisited, Phys.Rev. D79 (2009) 055023. arXiv:0902.0525, doi:10.1103/PhysRevD.79.055023.

[25] J. Romao, N. Rius, J. Valle, Supersymmetric signals in muon and $\tau$ decays, Nucl.Phys. B363 (1991) 369-384. doi:10.1016/0550-3213(91)80025-H.

[26] A. Jodidio, B. Balke, J. Carr, G. Gidal, K. Shinsky, et al., Search for Right-Handed Currents in Muon Decay, Phys.Rev. D34 (1986) 1967. doi:10.1103/PhysRevD.34.1967, 10.1103/PhysRevD.37.237.

[27] T. Hambye, Lepton flavor violation in low-scale seesaw models, in these proceedings. .

[28] A. Ilakovac, A. Pilaftsis, Flavor violating charged lepton decays in seesaw-type models, Nucl.Phys. B437 (1995) 491. arXiv:hepph/9403398, doi:10.1016/0550-3213(94)00567-X.

[29] J. Hisano, T. Moroi, K. Tobe, M. Yamaguchi, Lepton flavor violation via right-handed neutrino Yukawa couplings in supersymmetric standard model, Phys.Rev. D53 (1996) 2442-2459. arXiv:hep-ph/9510309, doi:10.1103/PhysRevD.53.2442.

[30] E. Arganda, M. J. Herrero, Testing supersymmetry with lepton flavor violating tau and mu decays, Phys.Rev. D73 (2006) 055003. arXiv:hep-ph/0510405, doi:10.1103/PhysRevD.73.055003.

[31] R. Mohapatra, J. Valle, Neutrino Mass and Baryon Number Nonconservation in Superstring Models, Phys.Rev. D34 (1986) 1642. doi:10.1103/PhysRevD.34.1642.

[32] A. Ilakovac, A. Pilaftsis, Supersymmetric Lepton Flavour Violation in Low-Scale Seesaw Models, Phys.Rev. D80 (2009) 091902. arXiv:0904.2381, doi:10.1103/PhysRevD.80.091902.

[33] R. Alonso, M. Dhen, M. Gavela, T. Hambye, Muon conversion to electron in nuclei in type-I seesaw models, JHEP 1301 (2013) 118. arXiv:1209.2679, doi:10.1007/JHEP01(2013)118.

[34] D. Dinh, A. Ibarra, E. Molinaro, S. Petcov, The $\mu-e$ Conversion in Nuclei, $\mu \rightarrow e \gamma, \mu \rightarrow 3 e$ Decays and TeV Scale See-Saw Scenarios of Neutrino Mass Generation, JHEP 1208 (2012) 125. arXiv:1205.4671, doi:10.1007/JHEP09(2013)023, 10.1007/JHEP08(2012)125.

[35] A. Ilakovac, A. Pilaftsis, L. Popov, Charged Lepton Flavour Violation in Supersymmetric Low-Scale Seesaw Models, Phys.Rev. D87 (2013) 053014. arXiv:1212.5939, doi:10.1103/PhysRevD.87.053014.

[36] M. Hirsch, F. Staub, A. Vicente, Enhancing $l_{i} \rightarrow 3 l_{j}$ with the $Z^{0}$-penguin, Phys.Rev. D85 (2012) 113013. arXiv:1202.1825, doi:10.1103/PhysRevD.85.113013.

[37] H. Dreiner, K. Nickel, F. Staub, A. Vicente, New bounds on trilinear R-parity violation from lepton flavor violating observables, Phys.Rev. D86 (2012) 015003. arXiv:1204.5925, doi:10.1103/PhysRevD.86.015003.

[38] M. Hirsch, W. Porod, L. Reichert, F. Staub, Phenomenology of the minimal supersymmetric $U(1)_{B-L} \times U(1)_{R}$ extension of the standard model, Phys.Rev. D86 (2012) 093018. arXiv:1206.3516, doi:10.1103/PhysRevD.86.093018.

[39] A. Abada, D. Das, A. Vicente, C. Weiland, Enhancing lepton flavour violation in the supersymmetric inverse seesaw beyond the dipole contribution, JHEP 1209 (2012) 015. arXiv:1206.6497, doi:10.1007/JHEP09(2012)015. 